\title{
NSMB at 25
}

This January 2018 issue starts the 25th year of NSMB's journey. We mark the occasion by launching a special series that celebrates the exciting research uncovering the fundamental principles behind biological processes.

n January 1994, the first issue of Nature Structural Biology was published, featuring an Editorial, three News and Views, one Correspondence, a Picture Story and nine Research Articles. At the time, it was but the second Nature research title (Nature Genetics was launched the previous year). Twentyfour years and over 3,600 Research Articles later, we begin volume 25 of the journal (our silver jubilee!) by reflecting on its trajectory and mission.

The inaugural editorial (https:// www.nature.com/articles/nsb0194-1. pdf) explained the genesis of the journal, alongside some rather accurate predictions of future structural achievements ("on everyone's wish list must be the ribosome, although that vast complex will take a little longer"). It also pointed to "the rapid growth of interest in the field", which remains as strong as ever today, as demonstrated by the so-called cryo-EM revolution, but also spurred by advances in X-ray crystallography (for example, lipidic cubic phase and XFEL), NMR and computational and integrative approaches. We should not overlook the role played by structural genomics; while those initiatives have received criticisms, their contributions to the field, in the form of new methodologies and pipelines for structural determination and a substantial number of new structures, cannot be denied.

The progress in structural biology has allowed us to explore more challenging targets, such as membrane proteins and large multisubunit complexes, with increasingly higher resolution and even to follow chemical reactions as they unfold. The sheer growth in the number of determined structures might have been difficult to foresee in early 1994, when they hovered around 1,600; there are now over 130,000 searchable structures in the wwPDB.

In 2004, the journal's title was changed to Nature Structural \& Molecular Biology (NSMB for short) to reflect its expanded scope as an integrated forum for research using multiple approachesnot only structural biology, but also biochemistry, molecular biology, genetics and so on-to obtain new mechanistic insight into biological processes. Confusion about NSMB's scope seems to linger to this day: some structural biologists feel that we are not interested in their work any longer, whereas non-structural biologists still think that structural data are required for a paper to be considered at NSMB. Both misconceptions can be dispelled if one scrolls through the Table of Contents of any recent issue of the journal.

What we want to publish is interesting and exciting biology revealed in molecular, if not atomic, detail. We are passionate about basic research that aims to decipher how cells and organisms 'work' at a fundamental level, how biological molecules are synthesized and organized and how they perform their functions. We champion scientific work dissecting the fundamental processes that drive life, such as DNA replication, transcription/translation and protein assembly, to name a few.

In our view, such basic knowledge is fascinating and worth pursuing in and of itself. It is also essential to understand how things go awry-for example, in cancer, infection and neurodegenerative diseases-to open avenues for intervention. Basic research provides the foundation to support medical and industrial applications, and we highlight the key role of structural information in driving drug design and engineering proteins and nucleic acids.

These ideals and principles form our core mission, and they are illustrated by the pieces we have commissioned for the "NSMB 25" anniversary series. We open with two Reviews in this issue. Hilger, Masureel and Kobilka explore the molecular bases of GPCR-mediated signaling, an important area of research with implications for drug development. The dynamic interplay of activated GPCRs with arrestin and with G protein underlies the cellular outcomes and the pharmacological properties of different agonists. Hasan, Sevvana, Kuhn and Rossmann review our current knowledge of the structural biology of Zika virus, which caused the 2015-2016 epidemic in South and Central America. While symptoms for most infected individuals are usually not serious, Zika virus infection has devastating consequences in pregnant women, as it can result in severe fetal brain defects.

We close with a heartfelt appreciation of the scientific communities for whom and with whom we work. Researchers play multiple paramount roles as NSMB partners, and for this, we are deeply grateful: to our authors, for entrusting their manuscripts in our hands, to our reviewers, for the thorough assessment and constructive comments they provide to help improve every paper, and to our readers, for their interest in what we publish and for not shying away from giving us candid feedback. We are proud to have made this journey with you at our side and look forward to the next 25 years!

Published online: 8 January 2018 https://doi.org/10.1038/s41594-017-0017-1 